\title{
Re-inventing yourself after spinal cord injury: a site-specific randomized clinical trial
}

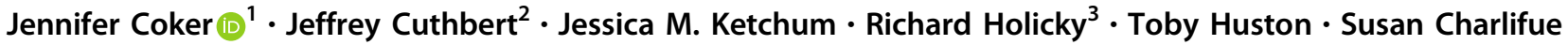

Received: 30 May 2018 / Accepted: 27 November 2018 / Published online: 20 December 2018

(c) International Spinal Cord Society 2018

\begin{abstract}
Study design Parallel-arm randomized controlled trial comparing an interactive group format versus a no-intervention control.

Objective To evaluate the efficacy of a replicable group treatment program for improving self-efficacy after spinal cord injury (SCI) in a single center cohort of adults.

Setting Regional SCI center.

Methods Participants included 81 individuals with SCI who were at least 4 weeks post-discharge from initial rehabilitation. Those randomized to treatment participated in six interactive learning sessions ( 2 hours each, one session per week) with structured and facilitated group interactions to improve self-efficacy. A wait-list control group was followed at matching time points for all outcome measures. The primary outcome measure was the Moorong Self-Efficacy Scale (MSES); secondary outcomes were the Generalized Self-Efficacy Scale (GSES); Diener Satisfaction with Life Scale (SWLS); Participation Assessment with Recombined Tools - Objective (PART-O); Patient Health Questionnaire - 9 (PHQ-9); and General Anxiety Disorder 7-item (GAD-7).

Results Individuals in the Treatment group had greater increases in MSES scores from baseline to immediately postintervention (6 weeks) than the Control group, but that difference did not remain significant after controlling for multiple comparisons. However, the improvement in the treatment group relative to the control group was not maintained through follow-up at 30 weeks. There was no evidence of an immediate or sustained treatment effect on any of the secondary outcomes.

Conclusions Self-efficacy improved for participants with SCI in the Treatment group, however, this improvement was not significant or maintained on follow-up.
\end{abstract}

\section{Introduction}

The consequences of spinal cord injury (SCI) can place significant demands on an individual's coping mechanisms and represent risk factors for development of symptoms such as depression and/or anxiety and decreased societal participation [1]. Previous research has described the prevalence of depressive disorders and anxiety symptoms following SCI between $11 \%$ and $60 \%$

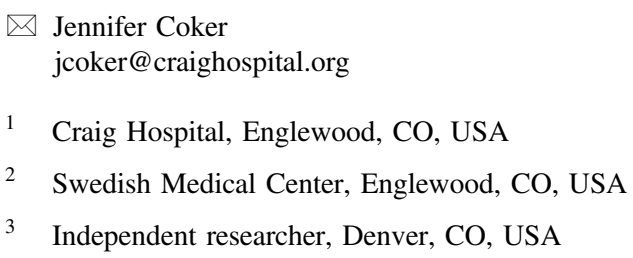

[2] and 20\% and 25\% respectively [3]. Interventions designed to promote psychological adjustment and adaptations are often included as a standard component of inpatient interdisciplinary rehabilitation programs [4]; however, following discharge from inpatient rehabilitation, many individuals with SCI do not receive ongoing counseling or education about psychological adjustment to disability. Furthermore, people with SCI indicate that their information needs are not met in many areas, with continuing stressors and challenges confronting them [5]. More effective post-acute treatment models are needed, not only to help individuals with SCI alleviate suffering from anxiety and depression symptoms, but also to build resilience to the stresses associated with a chronic physical disability and to enhance subjective well-being. 


\section{Self-efficacy, emotional distress, well being, and participation}

An individual's vulnerability and resilience to ongoing stresses associated with SCI are strongly influenced by personal beliefs about his/her capabilities for coping with them. People's beliefs about their capabilities for successfully performing tasks that affect their lives has been termed "self-efficacy" [6]. General self-efficacy is defined as "an individual's perception of his or her ability to perform across a variety of different situations" [7]. Efficacy beliefs form the basis for an individual's decision of what course of action to take, and the degree of effort to exert, when faced with an obstacle or challenge in life. A strong sense of general self-efficacy positively affects life choices, level of motivation, quality of functioning, resilience to adversity, and vulnerability to stress and depression [8]. In contrast, individuals with low self-efficacy tend to avoid challenges, discontinue tasks that are difficult, and be susceptible to higher levels of depression and stress, resulting in less satisfaction with life [6]. Studies of self-efficacy in those with SCI have been consistent with self-efficacy theory. People with SCI with low SCI-related self-efficacy beliefs have more anxiety and depression symptoms and lower quality of life [9].

\section{Enhancing self-efficacy beliefs after SCI}

Anxiety and depression symptoms may increase after completion of acute rehabilitation when the individual with SCI is faced with new limitations and challenges during community reintegration [1]. The association between low self-efficacy to emotional distress and reduced quality of life following SCI provides a theoretical basis for specifically targeting individuals with low self-efficacy for treatment aimed at strengthening their efficacy beliefs, rather than focused only at alleviating their anxiety and depression symptoms.

Cognitive behavioral therapy (CBT) is a treatment approach recommended for influencing self-efficacy beliefs of individuals with SCI [10]. CBT primarily is focused on changing thinking styles to facilitate emotional and behavioral change. Meta-analyses have found CBT to be effective for improving psychological outcomes following SCI including assertiveness, coping, self-efficacy, depression and quality of life, and adjustment $[3,11]$.

The purpose of this study was to evaluate the efficacy of a specific, replicable group CBT-based education intervention to enhance personal self-efficacy. We hypothesized that presenting positive psychotherapy topics in an interactive structured group format while restructuring maladaptive thought processes and providing experiential opportunities to reinforce behavioral change would result in an immediate treatment effect and a sustained treatment effect of increased self-efficacy, enhanced well-being, and improved societal participation. This intervention was specifically designed for a group setting, as this format provides an environment in which peer support can take place, and experiential learning can be undertaken and reinforced by both the individual and the peer group.

\section{Methods}

\section{Study intervention}

The intervention, Re-Inventing Yourself after SCI, is a replicable, manualized, educationally-based group therapeutic intervention program. This was a parallel-arm 1:1 randomized controlled clinical trial. The intervention consisted of six weekly facilitator-led sessions, with each session lasting $\sim 2$ hours. Table 1 provides a content outline of each intervention session.

Eight specific skills were presented over the course of the intervention to address reframing a person's method of looking at events, building confidence by focusing on

Table 1 Re-Inventing yourself after SCI: session content

Session 1: First Things First: Introductions and Identifying Goals

Overview of group sessions

Present key principle \#1: happiness formula

Present key principle \#2: reinvention ladder

Session 2: establish goals: prioritize and address needs

Present key principle \#3: smart goals

Individually set goals and review with group

Discuss the power of peer support

Session 3: reframing: how you think = how you feel

Review individual goals

Present key principle \#4: how thoughts drive emotions

Present key principle \#5: ABCDE approach (adversity, beliefs, consequences, disputing, energizing)

Session 4: overcoming barriers

Review key principle \#5 ABCDE approach focusing on D (disputing)

Discuss individual barriers that could hinder reaching goals

Present key principle \#6: untwisting thinking

Session 5: using character strengths

Identifying personal strengths

Present key principle \#7: using personal strengths

Practice problem solving using identified strengths

Session 6: gratitude and maintenance

Present key principle \#8: gratitude

Review personal goals using key principles 1-8

Discuss how to maintain the gains 
personal strengths, developing ways to recognize and appreciate the good in one's life, and expressing gratitude for positive attributes. These skills were presented in a specific sequence so that participants could gain mastery of introductory concepts before undertaking those that were more difficult and complex. The intervention sessions, led by group facilitators with extensive personal and professional experience in SCI rehabilitation (including a physical therapist, a nurse, and a social worker as well as an individual with $\mathrm{SCI}$ ), included didactic presentations of the key principles and experiential exercises such as goal setting and problem solving with extensive group discussion. At the end of each session, tasks were assigned to participants to be completed outside the group during the week between sessions; it was emphasized to participants that although these tasks were not mandatory, they would be useful and enhance understanding of course content and facilitate discussion in subsequent sessions (no data were collected on homework completion). Experiences from these activities and practice implementing the intervention principles were shared and discussed each week, providing additional opportunities for problem solving and feedback. A full description of the intervention and course content can be made available upon request.

All applicable institutional and governmental regulations concerning the ethical use of human volunteers were followed during the course of this research. This study was registered on ClinicalTrials.gov (Registration number: NCT01633164).

\section{Sample}

Community dwelling people with SCI living in the metropolitan and surrounding areas near a western US SCI rehabilitation hospital were contacted by mail and telephone regarding the study between October 2011 and November 2015. Individuals expressing interest were informed of the study in detail and screened by telephone to determine whether or not they met the following study inclusion criteria: history of traumatic or non-traumatic SCI at any level; at least 4 weeks post-discharge from initial inpatient rehabilitation; 18 years of age or older at the time of study enrollment; English speaking in order to complete study measures and participate in group interactions; and able to provide informed consent to participate.

Exclusion criteria included having a history of moderate or severe traumatic brain injury; current participation in another randomized controlled trial; living beyond a reasonable commuting distance from the study site; inability to verbally communicate; inability to attend group sessions; actively participating in another formal clinical group or psychological therapy; currently experiencing moderately severe or greater levels of depression which would require more intense treatment than provided in this intervention, as evidenced by a score of 15 or higher on the Personal Health Questionnaire-9 (PHQ-9) [12]; or currently self-efficacious, as determined by a score of 90 or higher on the Moorong Self-Efficacy Scale (MSES) [10]. Participants were recruited on a rolling basis throughout the course of the study; one to two groups were held per year.

\section{Procedures}

Participants meeting study criteria and providing informed consent completed the baseline assessment described below. Within each cohort, all participants completed baseline testing within 1 week of the treatment group beginning the intervention. Post-testing for both groups took place immediately following the completion of the 6week intervention, and follow-up assessments were completed at 8 week intervals post-intervention (14, 22, and 30 weeks post-enrollment). The control group received no intervention throughout the course of the study (there is no standard of care for self-efficacy therapy post-rehabilitation at our facility, thus no active control component of this study); however, they were tested at intervals identical to the treatment group. After data collection for each cohort was completed, control group participants were offered the intervention at no charge, but no further data collection was performed. A total of 81 participants were enrolled, with 81 completing the study (four participants in the treatment group completed baseline assessments only and thus were not followed, but were included in the intent-to-treat analysis). See Fig. 1 for the CONSORT diagram.

\section{Sample size and power analysis}

Preliminary data from a study examining the psychometric properties of the MSES in a sample of 108 community dwelling persons with SCI reported a mean MSES of 84.14 and a standard deviation of 21.72 [10]. These parameters were used, along with a varying estimate of the correlation among repeated measures $($ rho $=0.5-9)$. All sample size calculations were completed a priori using PASS 2008 assuming a two-group repeated measures design with five time points powered for the primary outcome MSES. Minimum sample sizes of 35 per group achieves at least $85 \%$ power at a $5 \%$ level of significance to detect a difference in MSES changes between groups of at least 16 points, assuming a standard deviation of 22, across all values of rho between 0.5 and 0.9. A 16-point difference on the MSES is equivalent to a change of one point of each question, and corresponds to a moderate effect size of $\sim 0.73$. We planned to enroll 85 participants to account for up to $18 \%$ attrition, to ensure a total sample size of at least 70 would have complete data at all time points for analysis. 
Assessed for eligibility $(n=200)$

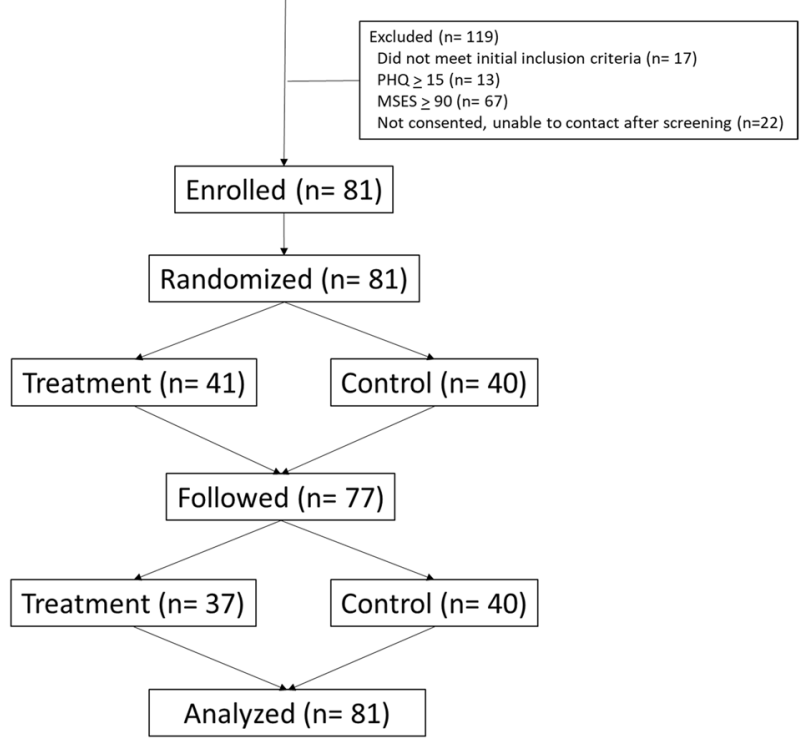

*There were four participants who withdrew prior to completion of the 6 weeks of treatment and thus were not followed; however, these participants were included in the intent-to-treat analysis.

Fig. 1 CONSORT diagram

\section{Randomization}

A group blocked randomization method was generated by the study statistician in cohorts of 17 participants, with participants in each cohort split into two groups (Treatment and Control) of 8 or 9 each. Participants were consented and enrolled prior to baseline assessment and randomized immediately post-baseline by the unblinded study coordinator who opened a sealed randomization envelope (sequentially numbered by block). Participants were notified of their allocation to treatment or control, and were asked to not share this information at any time with the research assistant who collected follow-up data. The blind was successfully maintained over the course of the study.

\section{Blinding}

Staff administering follow-up assessments were blinded to group assignment. Participants and group facilitators were carefully instructed not to reveal group assignment. The study statistician was also blinded.

\section{Baseline data collection}

The baseline interview consisted of a demographic questionnaire, an injury characteristics assessment, an online character strengths measure, and a battery of standardized outcome measures which was completed within 1 week prior to the onset of the intervention program. The online measure of character strengths, the Brief Strengths Test
[13], asks participants to rate how closely specific strengths and virtues match their personality. This measure was given only to those enrolled in the Treatment group at the baseline interview, the results of which were used throughout the intervention to make participants aware of areas of strength which can be utilized in day-to-day interactions and areas in need of development. Results of this measure were not included in any outcome analyses.

\section{Outcome measures}

The following outcome measures were collected at each assessment interval for both the Treatment and Control groups and used to determine the efficacy of the study intervention.

Primary outcome: The MSES is a 16-item self-report measure of self-efficacy related to everyday life activities, designed specifically for people with SCI. Individuals use a 7-point Likert scale ranging from 1 (very uncertain) to 7 (very certain) to rate their ability to perform 16 everyday tasks, with higher scores representing greater perceived selfefficacy [10]. A Total Score, ranging from 16 to 112 is calculated by summing all item responses. The MSES demonstrates excellent internal consistency $(\alpha=0.91-3)$, high reliability, and good convergent, concurrent, and divergent validity [14].

Secondary outcomes: the Generalized Self-Efficacy Scale (GSES) assesses a person's ability to cope with a variety of demands in life, with 10 items scored using a Likert rating scale, ranging from 1 (not at all true) to 4 (exactly true) [15]. This scale produces a Total Score ranging from 10-40, with higher scores indicating greater general self-efficacy [15]. The GSES has acceptable internal reliability and criterion validity [16], and has been validated in people with SCI [17].

The Diener Satisfaction with Life Scale (SWLS) measures global life satisfaction. This is a five item measure on which respondents rate their life satisfaction using a 7-point Likert scale, ranging from 1 (strongly disagree) to 7 (strongly agree) [18]. A Total Score, calculated by summing the items, ranges from 5 to 35 , with higher scores representing greater perceived quality of life. This measure has well-established psychometric properties, with high reliability, good content validity and criterion validity, and has been extensively used in research regarding people with SCI [19-21].

The Participation Assessment with Recombined Tools - Objective (PART-O) is a 17-item assessment of participation and has demonstrated good psychometric properties in measuring participation for people living with disabilities [22]. An Averaged Total Score is computed by taking the mean of 17 items, with scores ranging from 0 (never participate in these types of activities) to 5 (almost 
always participate in these types of activities), with higher scores representing greater participation. The PART-O has acceptable reliability and validity for individuals with SCI [23].

The PHQ-9 is a nine-item scale used to assess depression [12]. Respondents rate the frequency at which specific problems have been bothersome during the past 2 weeks, using a four point scale ranging from 0 (not at all) to 3 (nearly every day). A total score is calculated by summing all items (range $=0-29$ ), with higher scores indicating an increasing severity of depression [24]. This scale demonstrates excellent psychometrics, with excellent internal consistency, high test-retest reliability, and good criterion and construct validity, and has been used extensively in populations with SCI [12, 25-27].

The General Anxiety Disorder 7-item (GAD-7) is a seven-item measure that assesses the severity of general anxiety [28]. Using a four point Likert scale ranging from 0 (not at all sure) to 3 (nearly every day), respondents rate how often they have been bothered by specific symptoms during the past two weeks. A total severity score, ranging from 0 to 21 , is calculated by summing all items. This measure has demonstrated high internal consistency, good test-retest reliability, adequate convergent and discriminant validity, and has been used in previous studies involving people with SCI [28, 29].

\section{Treatment fidelity monitoring}

Fidelity assessments were completed with the intention of maintaining treatment adherence across groups. Group sessions were audio-recorded and scored by a study consultant to assess the degree to which the course material was covered and how well the group facilitators structured and guided the group process. Each recording was assigned a content and facilitation score using a five-point Likert scale, ranging from one $(<25 \%$ of course content covered; inappropriate amount of group facilitation) to five (80\% or more of course content covered; well-balanced group facilitation). Results of these reviews were shared with the group facilitators to provide feedback regarding the group facilitation process and, if necessary, to discuss alternative methods for delivering sparsely covered or difficult topics. However, feedback was not necessary as all of the sessions throughout the course of the study met the desired target for course content coverage.

\section{Statistical methods}

All data were analyzed using SAS version 9.4 [30] assuming a significance level of $5 \%(\alpha=0.05)$ unless otherwise noted. Sample characteristics were summarized by group using means/standard deviations (SDs) or medians/interquartile ranges (IQRs) for continuous variables and frequency counts and percentages for nominal variables. These characteristics were compared between the groups using $t$-tests and chi-square tests. Non-parametric alternatives (Kruskal-Wallis and Fisher's exact test) were used when distributional assumptions were not met.

The primary aim of this study was to assess changes in SCI-specific self-efficacy of individuals over time after participating in the Re-Inventing Yourself after SCI intervention compared to a group that has not received the intervention. The primary response variable for this study was the MSES Total Score for each individual at each of the five time points: baseline and at $6,14,22$, and 30 weeks (which corresponds to baseline, post-intervention and 8, 16, and 24 weeks postintervention). Data were analyzed as intent-to treat, using all available data from every case. A mixed-effects repeatedmeasures model was used to model and compare the changes in MSES scores over time between the groups. A first-order auto-regressive correlation structure was assumed to account for dependence in the repeated measures over time. The model included fixed effects for treatment, time, and the treatment by time interaction effect. In addition, the model adjusted for age, gender, race, time post-injury, and injury severity. Specific contrast statements were used to test if there was a significant treatment effect immediately postintervention at 6 weeks and if the treatment effect was maintained through the end of follow-up at 30 weeks by comparing the changes from baseline to 6 weeks and baseline to 30 weeks between the treatment and control groups. Within each model, a Bonferroni correction of $\alpha_{\mathrm{c}}=0.05 / 2=0.025$ was used to adjust for these two between group comparisons. Secondary outcomes include the GSES, SWLS, PHQ-9, GAD-7, and PART-O. Each of these outcomes was modeled and assessed using the same strategy as the primary outcome. Sensitivity analyses were conducted using models with (a) no covariates and (b) the last observation carried forward approach.

\section{Results}

Participants were recruited and enrolled between October 2011 and November 2015; data collection was complete in June 2016. The demographic and injury characteristic of the sample are summarized in Table 2 . On average, subjects were $\sim 50$ years old $(\mathrm{SD}=14.1)$, male $(82 \%)$, and Caucasian $(83 \%)$. The median time post injury was 86 months (interquartile range $=37.5-25.6$ ).

The estimated least squares mean MSES scores, adjusted for age, gender, race, time post-injury, and injury severity are summarized over time by group in Table 3 and shown in Fig. 2 (panel A). Changes in MSES over time within group and comparison of these changes between groups are 
Table 2 Demographic and injury characteristics by group

\begin{tabular}{lll}
\hline & $\begin{array}{l}\text { Treatment } \\
(n=41)\end{array}$ & $\begin{array}{l}\text { Control } \\
(n=40)\end{array}$ \\
\hline Age, mean (SD) & $48.0(12.8)$ & $52.0(15.3)$ \\
Months post injury, median (IQR) & $95.0(40-309)$ & $81.5(33-239)$ \\
Gender & & \\
Male, count (\%) & $34(82.9)$ & $32(80.0)$ \\
Female, count $(\%)$ & $7(17.1)$ & $8(20.0)$ \\
Race & & \\
White, count $(\%)$ & $32(78.0)$ & $35(87.5)$ \\
Not White, count $(\%)$ & $9(22.0)$ & $5(12.5)$ \\
Injury completeness & & $16(40.0)$ \\
Complete, count $(\%)$ & $19(46.3)$ & $24(60.0)$ \\
Incomplete, count $(\%)$ & $22(53.7)$ & \\
AIS & & $16(40.0)$ \\
A, count $(\%)$ & $19(46.3)$ & $3(7.5)$ \\
B, count $(\%)$ & $2(4.9)$ & $8(20.0)$ \\
C, count $(\%)$ & $7(17.1)$ & $13(32.5)$ \\
D, count $(\%)$ & $13(31.7)$ & $8(20.0)$ \\
Injury severity & & $11(27.5)$ \\
High tetra complete $(\%)$ & $7(17.1)$ & \\
Low tetra complete $(\%)$ & $9(22.0)$ & \\
Para complete $(\%)$ & $12(29.3)$ & \\
All Ds incomplete $(\%)$ & $13(31.7)$ & \\
\hline
\end{tabular}

$S D$ standard deviation, $I Q R$ interquartile range

summarized in Table 4. From baseline to 6 weeks (immediately post-intervention), there were significant increases in MSES of 4.68 in the Treatment group and nominal (nonsignificant) increases of 0.92 in the Control group. There was marginal evidence of a treatment effect $(\delta)$, with the Treatment group having greater improvements in MSES than the Control group by 3.76 from baseline to 6 weeks ( $p$ $=0.0341$ ), however this comparison was not significant after adjusting for multiple comparisons $\left(\alpha_{\mathrm{c}}=0.025\right)$. Furthermore, there was not statistical evidence that a treatment effect was sustained through 30 weeks of follow-up. Neither group showed significant changes in MSES from baseline to 30 weeks, nor was the comparison in changes between groups significant $(\delta=4.47, p=0.15)$. The conclusions of no significant treatment effect on MSES at 6 weeks or 30 weeks were consistent when modeling the data without adjusting for covariates $(0-6$ week $\delta=3.90, p=0.03$; 0 30 week $\delta=4.27, p=0.17$ ) and when using the last observation carried forward approach to handle missing data $(0-6$ week $\delta=2.67, p=0.15 ; 0-30$ week $\delta=4.48, p$ $=0.17$ ). A Cohen's d type estimate of effect size (ES) was computed as the estimated treatment effect divided by the baseline standard deviation (13.6), pooled across groups. The ESs at 6 weeks and 30 weeks were 0.3 and 0.4 , respectively, which are considered small to medium.
Analyses of the secondary outcomes are also summarized in Tables 3-4 and Fig. 2b-f. The Treatment group showed significant improvements in GSES, PHQ-9, and GAD-7 from baseline to 6 weeks whereas the Control group showed nominal improvements. Neither group showed significant changes in SWLS or PART-O from baseline to 6 weeks. Although the Treatment group exhibited nominally greater improvements in GSES, PHQ-9, and GAD-7 than the control group, comparison of these changes between groups were not significant, suggesting no real treatment effect on these outcomes at 6 weeks. Furthermore, there were no significant changes from baseline to 30 weeks follow-up for any of the secondary outcomes within either group, nor were the comparison in these changes significantly different between the groups. Conclusions remained the same when modeling outcomes without adjusting for covariates and when using the last observation carried forward approach. ESs at 6 and 30 weeks, respectively, were 0.30 and 0.40 for GSES, 0.12 and -0.02 for SWLS, -0.24 and -0.25 for PHQ- $9,-0.32$ and -0.41 for GAD-7, and 0.06 and 0.10 for PART-0, all considered small to medium.

\section{Discussion}

The purpose of this study was to evaluate the efficacy of a specific, replicable psychologically-based group CBT education intervention to enhance personal self-efficacy. We hypothesized that the intervention would result in an immediate treatment effect and a sustained treatment effect of increased self-efficacy, enhanced well-being, and improved societal participation. Results showed that participants in the Treatment group did not show significantly better improvements in outcomes than the Control group at 6 weeks or 30 weeks. There were significant group differences at baseline for SCI-specific and general self-efficacy, as well as anxiety, depression, and perceived quality of life; however, these were controlled for in the analyses by comparing the relative change at 6 and 30 weeks from baseline between the treatment groups. Baseline differences after randomization may be chance variation; we would not expect to see these differences in the long run and we do not believe the difference in MSES at baseline was substantial enough to have influenced the outcome of the study.

The a priori power analysis suggested that 35 subjects per group would be necessary to detect an effect size of 0.7 (based on a difference in mean changes of 16 units and a standard deviation of 22) with at least $85 \%$ power for correlations between 0.5 and 0.9 . Our study had slightly larger sample sizes $(N=41$ treatment and $N=40$ control), a substantially smaller standard deviation (13.7), and high correlation among the repeated measures $(\rho=0.85)$, resulting in higher power to detect smaller differences than 
A
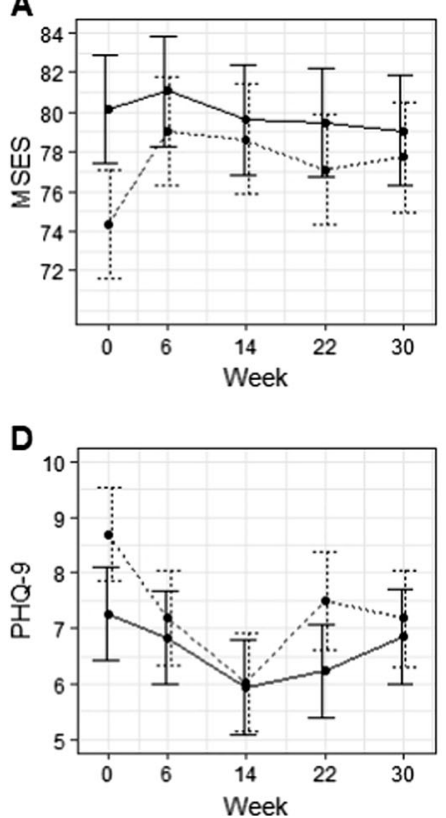

B

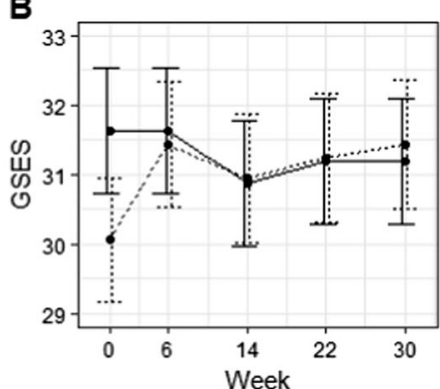

E

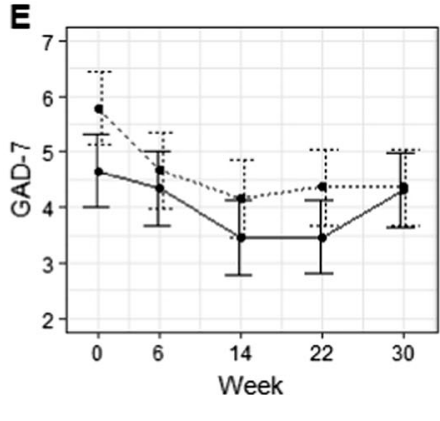

C

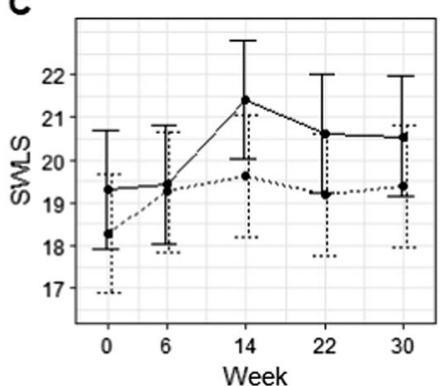

$\mathbf{F}$

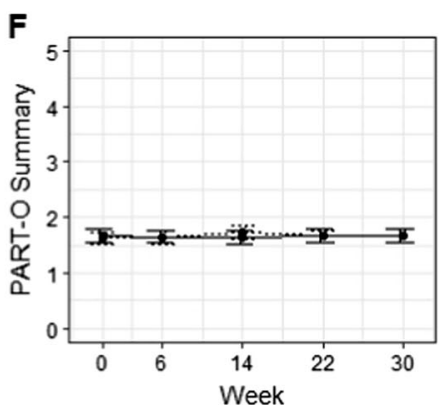

- Control $\cdots \cdot$... Treatment

Fig. 2 Changes in mean outcomes over time by group. Note: Error bars indicate standard deviations

anticipated. As such we saw a treatment effect of 3.76 marginally significant $(p=0.0341)$, which is considerably smaller than the 16-point difference we initially thought to be clinically meaningful. Based on communications with the instrument developer during the analysis, we believe that a 4-point difference may be clinically meaningful, but more research is needed.

Few attempts have been made to specifically enhance efficacy beliefs as mediators of therapeutic change for individuals with SCI. The intervention in the current study was a structured group CBT-based approach using positive psychotherapy concepts of positive emotion, engagement, relationships, purpose-meaning, and accomplishment to cognitively challenge, reframe, and restructure individuals' thoughts and beliefs about their intrinsic capabilities after SCI in order to enhance optimistic self-efficacy beliefs [3]. Group CBT has an advantage over individual therapy by providing the opportunity for sharing experiences that are common among members; group members provide support in a social environment where problems can be affirmed by a group of one's peers, failures can be readdressed, and success can be lauded [31, 32]. Mehta et al note many advantages of including CBT in a rehabilitation program, which are particularly relevant to the intervention we utilized. Among the advantages noted are that "it is structured, time limited, involves goal setting, engages clients, is skills based,..., can be delivered either on a group or individual basis, and is applicable to both inpatient and outpatient settings..." [3].

\section{Limitations}

The study design limited our ability to test for the impact of the intervention contents versus the group process alone versus the group process plus the intervention contents. Second, the follow-up time period may not have been adequate to assess sustainability of any gains appreciated in the intervention. A third limitation is generalizability of the study results to the population. Facilitators at the study site were involved with the development of and experienced in delivering the intervention so it is unknown if the intervention and results can be replicated. A multisite RCT of the intervention is currently underway to address all of these limitations. The mixed effects models utilized in this study assumed the outcome data were normally distributed. While no diagnostic issues were identified, the use of nonparametric statistical methods to analyze the ordinal outcome measures may have been more appropriate and led to different conclusions.

\section{Conclusions}

There was evidence of a significant treatment effect of the Re-Inventing Yourself After SCI intervention, with the Treatment group having significantly greater improvements in MSES than the Control group from baseline to 6 weeks. Even though the treatment effect was not significant after adjusting for multiple comparisons, the 4- 
Table 3 Estimated mean outcome by group and time

\begin{tabular}{|c|c|c|c|c|}
\hline Group & Time & Mean & SE & $95 \% \mathrm{CI}$ \\
\hline \multicolumn{5}{|c|}{ Moorong Self-Efficacy Scale (MSES) } \\
\hline \multirow[t]{3}{*}{ Treatment } & 0 Weeks (baseline) & 74.34 & 2.70 & $(68.98,79.71)$ \\
\hline & 6 Weeks (post-intervention) & 79.02 & 2.75 & $(73.57,84.47$ \\
\hline & 30 Weeks (follow-up) & 77.73 & 2.80 & $(72.18,83.28$ \\
\hline \multirow[t]{3}{*}{ Control } & 0 Weeks (aseline) & 80.16 & 2.75 & $(74.70,85.61)$ \\
\hline & 6 Weeks (post-intervention) & 81.07 & 2.76 & $(75.60,86.55$ \\
\hline & 30 Weeks (follow-up) & 79.07 & 2.76 & $(73.60,84.54$ \\
\hline \multicolumn{5}{|c|}{ Generalized Self-Efficacy Scale (GSES) } \\
\hline \multirow[t]{3}{*}{ Treatment } & 0 Weeks (baseline) & 30.06 & 0.89 & $(28.29,31.82$ \\
\hline & 6 Weeks (post-intervention) & 31.43 & 0.91 & $(29.63,33.24$ \\
\hline & 30 Weeks (follow-up) & 31.43 & 0.93 & $(29.59,33.27$ \\
\hline \multirow[t]{3}{*}{ Control } & 0 Weeks (baseline) & 31.64 & 0.90 & $(29.85,33.43$ \\
\hline & 6 Weeks (post-intervention) & 31.63 & 0.91 & $(29.84,33.43$ \\
\hline & 30 Weeks (follow-up) & 31.20 & 0.91 & $(29.40,33.00$ \\
\hline \multicolumn{5}{|c|}{ Satisfaction With Life Scale (SWLS) } \\
\hline \multirow[t]{3}{*}{ Treatment } & 0 Weeks (baseline) & 18.28 & 1.37 & $(15.56,20.99$ \\
\hline & 6 Weeks (post-intervention) & 19.25 & 1.40 & $(16.48,22.02$ \\
\hline & 30 Weeks (follow-up) & 19.38 & 1.43 & $(16.56,22.20$ \\
\hline \multirow[t]{3}{*}{ Control } & 0 Weeks (baseline) & 19.30 & 1.39 & $(16.55,22.05$ \\
\hline & 6 Weeks (post-intervention) & 19.43 & 1.39 & $(16.67,22.18$ \\
\hline & 30 Weeks (follow-up) & 20.56 & 1.39 & $(17.80,23.32$ \\
\hline \multicolumn{5}{|c|}{ Patient Health Questionnaire 9-Item (PHQ-9) } \\
\hline \multirow[t]{3}{*}{ Treatment } & 0 Weeks (baseline) & 8.70 & 0.84 & $(7.03,10.36)$ \\
\hline & 6 Weeks (post-intervention) & 7.19 & 0.87 & $(5.48,8.90)$ \\
\hline & 30 Weeks (follow-up) & 7.18 & 0.88 & $(5.44,8.91)$ \\
\hline \multirow[t]{3}{*}{ Control } & 0 Weeks (baseline) & 7.26 & 0.84 & $(5.59,8.93)$ \\
\hline & 6 Weeks (post-intervention) & 6.83 & 0.85 & $(5.16,8.51)$ \\
\hline & 30 Weeks (follow-up) & 6.85 & 0.85 & $(5.18,8.53)$ \\
\hline \multicolumn{5}{|c|}{ General Anxiety Disorder 7-Item (GAD-7) } \\
\hline \multirow[t]{3}{*}{ Treatment } & 0 Weeks (baseline) & 5.79 & 0.66 & $(4.49,7.08)$ \\
\hline & 6 Weeks (post-intervention) & 4.67 & 0.68 & $(3.32,6.01)$ \\
\hline & 30 Weeks (follow-up) & 4.36 & 0.69 & $(3.00,5.73)$ \\
\hline \multirow[t]{3}{*}{ Control } & 0 Weeks (baseline) & 4.66 & 0.66 & $(3.35,5.97)$ \\
\hline & 6 Weeks (post-intervention) & 4.33 & 0.67 & $(3.01,5.66)$ \\
\hline & 30 Weeks (follow-up) & 4.32 & 0.67 & $(3.00,5.64)$ \\
\hline \multicolumn{5}{|c|}{ Participant Assessment with Recombined Tools - objective (PART-O) } \\
\hline \multirow[t]{3}{*}{ Treatment } & 0 Weeks (baseline) & 1.63 & 0.12 & $(1.39,1.86)$ \\
\hline & 6 Weeks (post-intervention) & 1.64 & 0.12 & $(1.40,1.88)$ \\
\hline & 30 Weeks (follow-up) & 1.68 & 0.12 & $(1.44,1.92)$ \\
\hline \multirow[t]{3}{*}{ Control } & 0 Weeks (baseline) & 1.67 & 0.12 & $(1.44,1.91)$ \\
\hline & 6 Weeks (post-intervention) & 1.65 & 0.12 & $(1.42,1.89)$ \\
\hline & 30 Weeks (follow-up) & 1.67 & 0.12 & $(1.43,1.91)$ \\
\hline
\end{tabular}

SE standard error, $C I$ confidence interval point difference between the groups may be clinically meaningful (based on personal communications with the instrument developer). While there was evidence of a significant treatment effect on MSES immediately postintervention, there was not statistical evidence that the treatment effect was sustained through 30 weeks of follow-up. Neither the treatment nor the control group showed significant changes in MSES from baseline to 30 weeks, nor was the comparison in this change significantly different between groups. 
Table 4 Changes in outcomes over time by group and comparison of the changes between groups

\begin{tabular}{|c|c|c|c|c|c|}
\hline & & Mean & SE & $95 \% \mathrm{CI}$ & $p$-value \\
\hline \multicolumn{6}{|c|}{ Moorong Self-Efficacy Scale (MSES) } \\
\hline \multirow[t]{3}{*}{6 Weeks -0 Weeks } & Treatment & 4.68 & 1.27 & $(2.17,7.19)$ & 0.0003 \\
\hline & Control & 0.92 & 1.22 & $(-1.49,3.32)$ & 0.4544 \\
\hline & Treatment - control & 3.76 & 1.77 & $(0.28,7.24)$ & 0.0341 \\
\hline \multirow[t]{3}{*}{30 Weeks - 0 Weeks } & Treatment & 3.39 & 2.22 & $(-0.97,7.75)$ & 0.1276 \\
\hline & Control & -1.09 & 2.16 & $(-5.34,3.16)$ & 0.6149 \\
\hline & Treatment - control & 4.47 & 3.10 & $(-1.61,10.56)$ & 0.1492 \\
\hline \multicolumn{6}{|c|}{ Generalized Self-Efficacy Scale (GSES) } \\
\hline \multirow[t]{3}{*}{6 Weeks - 0 Weeks } & Treatment & 1.38 & 0.51 & $(0.37,2.38)$ & 0.0075 \\
\hline & Control & 0.00 & 0.49 & $(-0.96,0.96)$ & 0.9922 \\
\hline & Treatment - control & 1.38 & 0.71 & $(-0.01,2.77)$ & 0.0517 \\
\hline \multirow[t]{3}{*}{30 Weeks - 0 Weeks } & Treatment & 1.37 & 0.84 & $(-0.29,3.03)$ & 0.1054 \\
\hline & Control & -0.44 & 0.82 & $(-2.06,1.18)$ & 0.5924 \\
\hline & Treatment - control & 1.81 & 1.18 & $(-0.51,4.13)$ & 0.1254 \\
\hline \multicolumn{6}{|c|}{ Satisfaction With Life Scale (SWLS) } \\
\hline \multirow[t]{3}{*}{6 Weeks - 0 Weeks } & Treatment & 0.98 & 0.80 & $(-0.60,2.55)$ & 0.2235 \\
\hline & Control & 0.12 & 0.75 & $(-1.36,1.60)$ & 0.8688 \\
\hline & Treatment - control & 0.85 & 1.10 & $(-1.31,3.01)$ & 0.4391 \\
\hline \multirow[t]{3}{*}{30 Weeks - 0 Weeks } & Treatment & 1.10 & 1.31 & $(1.47,3.67)$ & 0.3999 \\
\hline & Control & 1.26 & 1.27 & $(-1.24,3.75)$ & 0.3229 \\
\hline & Treatment - control & -0.16 & 1.82 & $(-3.74,3.43)$ & 0.9322 \\
\hline \multicolumn{6}{|c|}{ Patient Health Questionnaire 9-Item (PHQ-9) } \\
\hline \multirow[t]{3}{*}{6 Weeks -0 Weeks } & Treatment & -1.51 & 0.66 & $(-2.80,-0.21)$ & 0.0226 \\
\hline & Control & -0.43 & 0.62 & $(-1.65,0.80)$ & 0.4943 \\
\hline & Treatment - control & -1.08 & 0.91 & $(-2.87,0.70)$ & 0.2328 \\
\hline \multirow[t]{3}{*}{30 Weeks - 0 Weeks } & Treatment & -1.52 & 0.95 & $(-3.39,0.35)$ & 0.1117 \\
\hline & Control & -0.41 & 0.93 & $(-2.23,1.42)$ & 0.6600 \\
\hline & Treatment - control & -1.11 & 1.33 & $(-3.72,1.50)$ & 0.4042 \\
\hline \multicolumn{6}{|c|}{ General Anxiety Disorder 7-Item (GAD-7) } \\
\hline \multirow[t]{3}{*}{6 Weeks - 0 Weeks } & Treatment & -1.12 & 0.52 & $(-2.14,-0.10)$ & 0.0322 \\
\hline & Control & -0.32 & 0.50 & $(-1.31,0.67)$ & 0.5207 \\
\hline & Treatment - control & -0.80 & 0.72 & $(-2.22,0.63)$ & 0.2706 \\
\hline \multirow[t]{3}{*}{30 Weeks - 0 Weeks } & Treatment & -1.42 & 0.75 & $(-2.90,0.06)$ & 0.0598 \\
\hline & Control & -0.34 & 0.74 & $(-1.78,1.11)$ & 0.6481 \\
\hline & Treatment - control & -1.08 & 1.05 & $(-3.16,0.99)$ & 0.3033 \\
\hline \multicolumn{6}{|c|}{ Participant Assessment with Recombined Tools - Objective (PART-O) } \\
\hline \multirow[t]{3}{*}{6 Weeks -0 Weeks } & Treatment & 0.02 & 0.05 & $(-0.08,0.12)$ & 0.7425 \\
\hline & Control & -0.02 & 0.05 & $(-0.11,0.08)$ & 0.7003 \\
\hline & Treatment - control & 0.04 & 0.07 & $(-0.10,0.17)$ & 0.6147 \\
\hline \multirow[t]{3}{*}{30 Weeks - 0 Weeks } & Treatment & 0.05 & 0.09 & $(-0.12,0.23)$ & 0.5471 \\
\hline & Control & 0.00 & 0.09 & $(-0.17,0.17)$ & 0.9643 \\
\hline & Treatment - control & 0.06 & 0.12 & $(-0.19,0.30)$ & 0.6432 \\
\hline
\end{tabular}

$S E$ standard error, $C I$ confidence interval
As described above in the study limitations, it is unclear from this study whether the contents of the intervention itself, the group process alone, or a combination of both resulted in initial improvements in the primary and selected secondary outcomes. A new RCT of this intervention is currently underway that adds a third study arm in which participants receive the intervention by video, removing the effects of the group interaction. In addition, although there was no statistical evidence for sustained improvements in outcomes, the length of follow-up is being expanded to 
determine if any gains appreciated during the intervention stabilize, improve, or decline over a longer period of time.

\section{Data archiving}

There are no data to deposit at this time. Reasonable requests for data will be considered.

Acknowledgements We thank Sara Huey and Stephanie Bennett Maunton, Research Assistants, who performed all data collection for this study. We thank the group facilitators; Terry Chase, Kristi Staniszewski, Nicole Miller, and Rachel Wilson, as well as Jody Newman who assessed facilitator fidelity. We sincerely thank all of the participants for their time and involvement.

Funding This study was funded by the National Institute on Disability and Rehabilitation Research/National Institute on Disability, Independent Living, and Rehabilitation Research, Grant \# H133N110006/905SI5003.

Author contributions JC was responsible for adherence to the protocol during data collection, interpreting results, and writing and revising the manuscript. JC was responsible for designing the protocol, oversight of the study during data collection, and interpreting the results. JK was responsible for analyzing data and interpreting results. RH was responsible for designing the intervention and interpreting results. $\mathrm{TH}$ was responsible for designing the intervention and protocol and interpreting results. SC was responsible for designing the protocol, interpreting results, and writing the manuscript. All authors helped shape the manuscript and provided critical feedback during revisions.

\section{Compliance with ethical standards}

Conflict of interest The authors declare that they have no conflict of interest.

Ethical approval We certify that all applicable institutional and governmental regulations concerning the ethical use of human volunteers were followed during the course of this research.

\section{References}

1. Middleton J, Craig A. Psychological challenges in treating persons with spinal cord injury. In: Craig A, Tran Y, editors. Psychological dynamics associated with spinal cord injury rehabilitation: New directions and best evidence. New York: Nova Science Publishers; 2008. p. 3-53.

2. Craig A, Tran Y, Middleton J. Psychological morbidity and spinal cord injury: a systematic review. Spinal Cord. 2009;47:108-14.

3. Mehta S, Orenczuk S, Hansen K, Aubut J, Hitzig S, Legassic M, et al. An evidence-based review of the effectiveness of cognitive behavioral therapy for psychosocial issues post-spinal cord injury. Rehabil Psychol. 2011;56:15-25.

4. Huston T, Gassaway J, Wilson C, Gordon S, Koval J, Schwebel A. The SCIRehab project: treatment time spent in SCI rehabilitation. Psychology treatment time during inpatient spinal cord injury rehabilitation. J Spinal Cord Med. 2011;34:196-204.

5. Gontkovsky ST, Russum P, Stokic DS. Perceived information needs of community-dwelling persons with chronic spinal cord injury: findings of a survey and impact of race. Disabil Rehabil. 2007;29:1305-12.
6. Bandura A. Social learning theory. Oxford, England: PrenticeHall; 1977. 247 p.

7. Luszczynska A, Scholz U, Schwarzer R. The General SelfEfficacy Scale: Multicultural validation. Stud J Psychol 2005;129:439-57.

8. Bandura A. Self-efficacy. In: Ramachaudran VS, editor. Encyclopedia of human behavior. 4. New York, NY: Academic Press; 1994. p. 71-81.

9. Horn W, Yoels W, Wallace D, Macrina D, Wrigley M. Determinants of self-efficacy among persons with spinal cord injuries. Disabil Rehabil. 1998;20:138-41.

10. Middleton JW, Tate RL, Geraghty TJ. Self-efficacy and spinal cord injury: psychometric properties of a new scale. Rehab Psychol. 2003;48:281-7.

11. Dorstyn D, Mathias J, Denson L. Efficacy of cognitive behavioral therapy for the management of psychological outcomes following spinal cord injury: a meta-analysis. J Health Psychol. 2011;16:374-91.

12. Kroenke K, Spitzer RL, Williams JB. The PHQ-9: validity of a brief depression severity measure. $\mathrm{J}$ Gen Intern Med. 2001;16:606-13.

13. University_of_Pennsylvania. The Brief Strengths Test 2011 [Available from: http://www.authentichappiness.sas.upenn.edu/ questionnaires.aspx.

14. Miller SM. The measurement of self-efficacy in persons with spinal cord injury: psychometric validation of the moorong selfefficacy scale. Disabil Rehabil. 2009;31:988-93.

15. Schwarzer R, Jerusalem M. Generalized self-efficacy scale. In: Weinman J, Wright S, Johnston M, editors. Measures in health psychology: A user's portfolio Causal and control beliefs. Windsor, UK: NFER-NELSON; 1995. p. 35-7.

16. Schwarzer R. The General Self-Efficacy Scale: Freie Universität Berlin, Psychologie; 2011 [psychometric review of the GSES].

17. Ljungberg I, Kroll T, Libin A, Gordon S. Using peer mentoring for people with spinal cord injury to enhance self-efficacy beliefs and prevent medical complications. J Clin Nurs. 2011;20:351-8.

18. Diener E, Emmons RA, Larsen RJ, Griffin S. The satisfaction with life scale. J Pers Assess. 1985;49:71-5.

19. Charlifue S, Apple D, Burns SP, Chen D, Cuthbert JP, Donovan $\mathrm{WH}$, et al. Mechanical ventilation, health, and quality of life following spinal cord injury. Arch Phys Med Rehabil. 2011;92:457-63.

20. Hetz SP, Latimer AE, Arbour-Nicitopoulos KP, Martin Ginis KA. Secondary complications and subjective well-being in individuals with chronic spinal cord injury: associations with self-reported adiposity. Spinal Cord. 2011;49:266-72.

21. Pavot W, Diener E, Colvin CR, Sandvik E. Further validation of the Satisfaction with Life Scale: evidence for the cross-method convergence of well-being measures. J Pers Assess. 1991;57:149_ 61.

22. Whiteneck GG, Dijkers MP, Heinemann AW, Bogner JA, Bushnik T, Cicerone KD, et al. Development of the participation assessment with recombined tools-objective for use after traumatic brain injury. Arch Phys Med Rehabil. 2011;92:542-51.

23. Bogner JA, Whiteneck GG, Corrigan JD, Lai JS, Dijkers MP, Heinemann AW. Comparison of scoring methods for the participation assessment with recombined tools-objective. Arch Phys Med Rehabil. 2011;92:552-63.

24. Spitzer RL, Kroenke K, Williams JBW. Validation and utility of a self-report version of PRIME-MD: the PHQ primary care study. Primary Care Evaluation of Mental Disorders. Patient Health Questionnaire. JAMA. 1999;282:1737-44.

25. Fann J, Bombardier C, Richards J, Tate R, Wilson C, Temkin N. Depression after spinal cord injury: comorbidities, mental health service use, and adequacy of treatment. Arch Phys Med Rehabil. 2009;92:352-60. 
26. Martin A, Rief W, Klaiberg A, Braehler E. Validity of the Brief Patient Health Questionnaire Mood Scale (PHQ-9) in the general population. Gen Hosp Psychiatry. 2006;28:71-7.

27. Spitzer RL, Williams JB, Kroenke K. Validity and utility of the Patient Health Questionnaire in assessment of 3000 obstetric-gynecologic patients: the PRIME-MD Patient Health Questionnaire ObstetricsGynecology Study. Am J Obstet Gynecol. 2000;183:759-69.

28. Spitzer RL, Kroenke K, Williams JB, Lowe B, Spitzer RL, Kroenke K, et al. A brief measure for assessing generalized anxiety disorder: the GAD-7. Arch Int Med. 2006;166:1092-7.

29. ClinicalTrials.gov. Treatment of Mild to Moderate Depression Symptoms in Patients With Spinal Cord Injury Bethesda
(MD) National Library of Medicine (US); 2008. cited Identifier: NDNO60032SS. Identifier NCT00287391. Available at: http:// clinicaltrialsfeeds.org/clinical-trials/show/NCT00735670.

30. SAS Institute Inc. SAS 9.3 ed. Cary, NC: SAS Institute Inc; 2011.

31. Leszcz M, Yalom ID, Norden M. The value of inpatient group psychotherapy: patients' perceptions. Int J Group Psychother. 1985;35:411-33.

32. Manassis K, Mendlowitz SL, Scapillato D, Avery D, Fiksenbaum L, Freire M, et al. Group and individual cognitivebehavioral therapy for childhood anxiety disorders: a randomized trial. J Am Acad Child Adolesc Psychiatry. 2002; 41:1423-30. 